\title{
EACHL Effect on Esophageal Carcinoma Cells and the Interaction with Bovine Serum Albumin
}

\author{
Fuchun $\mathrm{SI}^{1, \mathrm{a}, *}$ \\ Laboratory of molecular biology for TCM \\ Henan University of Chinese Medicine \\ Zhengzhou 450046, China \\ a e-mail: sifc2000@hotmail.com
}

\author{
Wenbin WANG ${ }^{1,2, b}$ \\ ${ }^{1}$ Laboratory of molecular biology for TCM \\ Henan University of Chinese Medicine \\ Zhengzhou 450046, China \\ ${ }^{2}$ National Key Laboratory of Cryospheric Sciences/Tien \\ Shan Glaciological Station, Cold and Arid Regions \\ Environmental and Engineering Research Institute, \\ Chinese Academy of Sciences, Lanzhou 730000, China \\ be-mail: wwellbin@126.com
}

\begin{abstract}
EACHL was extracted from the Chinese honey locust. MTT assay was used to test the cytotoxicity of the extracts on esophageal carcinoma cells. The result of MTT assay showed the Ic50 values of Eca109 and TE1 are $11.53,32.07 \mu \mathrm{g} \cdot \mathrm{ml}^{-1}$, respectively. The interaction of EACHL with bovine serum albumin (BSA) was studied at pH 7.40 (ionic strength 0.10 $\mathrm{mol} \cdot \mathrm{L}^{-1}$ ) by fluorescence. EACHL can quench the intrinsic fluorescence of BSA, the quenching mechanism is static quenching. The thermodynamic parameters, $\Delta H(-1175.43$ $\left.\mathrm{kJ} \cdot \mathrm{mol}^{-1}\right), \Delta \mathrm{S}\left(-4035.13 \mathrm{~J} \cdot \mathrm{mol}^{-1} \cdot \mathrm{K}^{-1}\right)$ and $\Delta \mathrm{G}(-47.22$ to -11.99 $\left.\mathrm{kJ} \cdot \mathrm{mol}^{-1}\right)$, indicated that the Hydrogen bonding and van der Waals force played major role. The binding constant $\left(K_{b}\right)$ were $1.3816 \times 10^{8} \mathrm{~L} \cdot \mathrm{mol}^{-1}(303 \mathrm{~K})$ and $1.0010 \times 10^{2} \mathrm{~L} \cdot \mathrm{mol}^{-1}(313 \mathrm{~K})$. Synchronous fluorescence studies showed that the conformation of BSA could be changed during EACHL was added.
\end{abstract}

Keywords-Chinese honey locus; Esophageal carcinoma cell; MTT; Bovine serum albumin

\section{INTRODUCTION}

Worldwide esophageal carcinoma is the sixth leading cause of cancer $\left(406,800\right.$ death cases) ${ }^{[1]}$. In men, the incidence is highest in China. In recent 15 years, we have carried out purposeful studies of anti-esophageal carcinoma medicine from over 500 kinds Chinese traditional herbs, found that the Chinese honey locust is one of the most effective drugs for the treatment of esophageal carcinoma ${ }^{[2]}$. Chinese honey locust (the seeds of Gleditsia sinensis) belongs to the Caesalpinioideae family ${ }^{[3]}$, which is widely used on Esophageal cancer. To find the active substance of the Chinese honey locust, the extracts were obtained by the technology of solvent-refining. And then the ethyl acetate fraction subjected to Dianion HP-20 column and silica gel, further purified with High performance liquid chromatograph (HPLC) and many times recrystallization, obtained light yellow amorphous solid powder (EACHL). By MTT assay, the ethyl acetate and n-butanol fractions showed good cytotoxic activity to the esophageal carcinoma cells (Eca109 and TE1).

Bovine Serum Albumin (BSA) is the most abundant soluble protein in the blood plasma, and can help to keep colloidal osmotic blood pressure and act as the transportation and disposition of many drugs, and is capable of binding, delivering an extraordinarily diverse range of endogenous and exogenous compounds, through the bloodstream to their target organs ${ }^{[4]}$. Investigations the interaction between the active ingredient of traditional Chinese medicine and BSA is helpful for explaining the medicine mechanism. It is especially useful for select anti-medicine vitro, and can giving theory guide for anti-medicine design ${ }^{[5]}$.

In this paper, the active ingredients (EACHL) were extracted from the Chinese honey locust. The cytotoxicity of the EACHL on two esophageal carcinoma cells was tested by MTT assay. The interaction mechanism of EACHL with BSA was investigated under physiological conditions by spectrofluorimetry. The binding parameters, thermodynamic functions for binding reaction (at $303 \mathrm{~K}$ and $313 \mathrm{~K}$ ) were calculated.

\section{EXPERIMENTAL}

\section{A. Apparatus and Reagents}

F-7000 fluorescence spectrophotometer (Hitachi, Japan), pH213 acidity meter (Portugal).

Dianion HP-20 (Mitsubishi), silica gel GF254 (BR), Eca109 and TE1 cells (Department of Medical Oncology, Cancer Institute \&Hospital, Chinese Academy of Medical Sciences), Bovine serum albumin, BSA (Beijing Olympic nemesis Biological Technology Co. Ltd.China, relative molecular mass 65000), was dissolved daily in $0.1 \mathrm{~mol} / \mathrm{L}$ Tris- $\mathrm{HCl}$ buffer solution $(\mathrm{pH}=7.3$ ) to prepare a solution $(1 \times 10-5 \mathrm{~mol} / \mathrm{L})$, EACHL to prepare a solution $(80 \mathrm{mg} / \mathrm{L})$, All chemicals were analytical-reagent grade. All solutions were prepared with double-distilled water. 


\section{B. Methods}

Fluorescence spectra were carried out on a Hitachi F-7000 fluorescence spectrophotometer using $1.0 \mathrm{~cm}$ quartz cells. The excitation wavelength of BSA studied in this work was $280 \mathrm{~nm}$.

\section{RESULTS AND DISCUSSION}

\section{Extracting Active Ingredient}

The technology of solvent-refining was used to extract the active fractions. The Chinese honey locust was immersed in $95 \%$ ethanol for 5 days at room temperature. The crude extraction was dried and suspended in water and successively treated with chloroform, ethyl acetate and n-butanol. Then, the ethyl acetate fraction subjected to Dianion HP-20 column and successively eluted with $\mathrm{H} 2 \mathrm{O}, 30 \%, 50 \%, 70 \%, 90 \%$ (v/v) EtOH-H2O, respectively. The $50 \% \mathrm{EtOH}$ fraction was chromatographed on silica gel. Subfraction was subjected to repeating column chromatography on silica geland, further purified by HPLC and with many times recrystallization, obtained light yellow amorphous solid powder (EACHL).

\section{MTT Assay Antitumor Activities}

The in vitro The cytotoxic effect of the EACHL on Eca109 and TE1 have been determined by the MTT assay, the EACHL exhibits inhibition on Eca109 and TE1 cancer cells with IC50 of $11.53,32.07 \mu \mathrm{g} \cdot \mathrm{ml}-1$, respectively. The EACHL has potential application in treatment esophageal carcinoma.

\section{E. Fluorescence of BSA Was Quenched By EACHL}

BSA has intrinsic fluorescence due to the presence of amino acids, mainly tryptophan (Trp) and tyrosine (Tyr) residues. So, fluorescence technique is wide spread application in investigating of interactions between the active ingredient of Chinese herb and protein molecules [6]. The interaction between EACHL and BSA has been monitored upon exciting BSA at $280 \mathrm{~nm}$ where both Trp and Tyr residues get excited. The variations of emission spectra were shown in Figure 1. The fluorescence quenching spectra of solutions containing a BSA fixed concentration and different concentrations of EACHL at $303 \mathrm{~K}$ and $313 \mathrm{~K}$, It can be observed that the fluorescence intensity of BSA decreases regularly with the increase addition of EACHL, under the experimental condition, the EACHL does not produce any emission in the specified range of study, and there is no significant emission wavelength shift. These results suggest that EACHL interact with BSA can quench BSA intrinsic fluorescence.
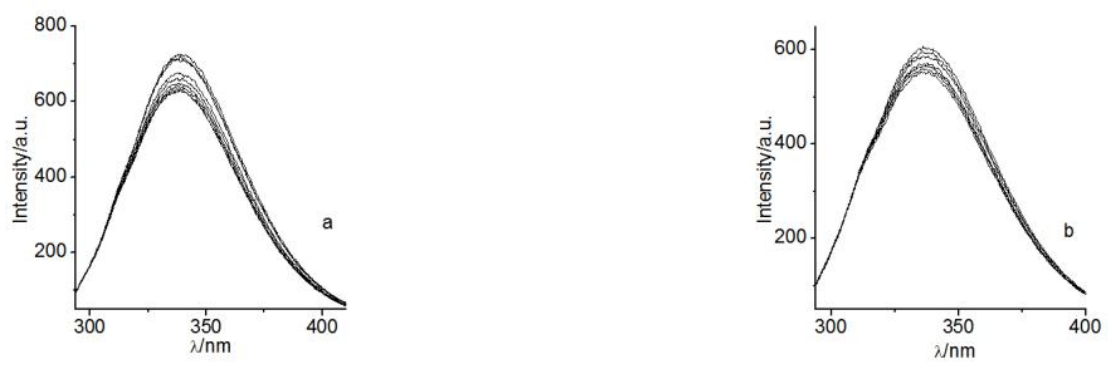

Figure 1. Changes in the emission spectra $(\lambda \mathrm{ex}=280 \mathrm{~nm})$ of the BSA $\left(1.0 \times 10^{-5} \mathrm{~mol} / \mathrm{L}\right)$ upon increasing concentrations of EACHL (a) $303 \mathrm{~K}$ and (b) $313 \mathrm{~K}$

\section{F. Quenching Mechanism}

Static and dynamic quenching are two quenching types in characterizing the mechanism of the interaction between quenchers and macromolecules. Static quenching refers to the formation of a non-fluorescence fluorophore-quencher complex. With temperature increasing, the stability of fluorophore-quencher complex reduced, the quenching constant decreased. Dynamic quenching refers to the quencher diffusing towards the fluorophore during the lifetime of the excited state and, upon contact, the fluorophore returns to the ground state without emission of a photon. Dynamic quenching mechanism was probed using the Stern-Volmer equation:

$$
F_{0} / F=1+K_{q} \tau_{0}[\mathrm{Q}]=1+K_{\mathrm{sv}}[\mathrm{Q}]
$$

Where $\mathrm{F} 0$ and $\mathrm{F}$ represent the fluorescence intensities of BSA in the absence and in the presence of the external quencher, $\mathrm{Kq}$ is the bimolecular quenching rate constant, $\mathrm{KSV}$ is the Stern-Volmer quenching constant, $\tau 0$ is the average lifetime of biopolymers without quencher, the fluorescence lifetime of the biopolymers is $10-8 \mathrm{~s},[\mathrm{Q}]$ is the concentration of the quencher.

The plot of $\mathrm{F} 0 / \mathrm{F}$ against [Q] at different temperature shown in Fig. 2. 

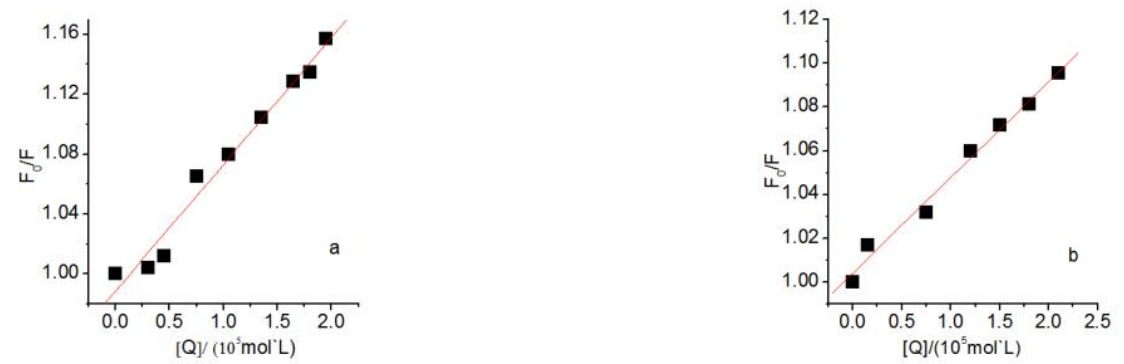

Figure 2. Stern-Volmer plot for the quenching of BSA by EACHL at different temperatures (a) $303 \mathrm{~K}$ and (b) $313 \mathrm{~K}$

Equation (1) was applied to determine KSV by linear regression from a plot of $\mathrm{F} 0 / \mathrm{F}$ against $[\mathrm{Q}]$, hence, Kq can be caculate by $\mathrm{Kq}=\mathrm{KSV} / \tau 0$. As shown in Table I.

TABLE I. CONSTANT OF THE SYSTEMS OF INTERACTION BETWEEN EACHL AND BSA AT DIFFERENT TEMPERATURES

\begin{tabular}{|c|c|c|}
\hline $\mathrm{T} /(\mathrm{K})$ & $K_{\mathrm{sv}} /\left({\left.\mathrm{L} \cdot \mathrm{mol}^{-1}\right)}^{-1}\right.$ & $K_{\mathrm{q}} /\left(\mathrm{L} \cdot \mathrm{mol}^{-1} \cdot \mathrm{s}^{-1}\right)$ \\
\hline 303 & $8.454 \times 10^{3}$ & $8.454 \times 10^{11}$ \\
\hline 313 & $4.364 \times 10^{3}$ & $4.364 \times 10^{11}$ \\
\hline
\end{tabular}

For the EACHL-BSA systems, the values of KSV and Kq $=\mathrm{KSV} / \tau 0$ obtained from the plots at $303 \mathrm{~K}$ and $313 \mathrm{~K}$ are shown in Table 1. According to the literatures ${ }^{[7,8]}$, which suggest that the fluorescence quenching mechanism is mainly static quenching.

G. The Binding Constant and The Binding Sites

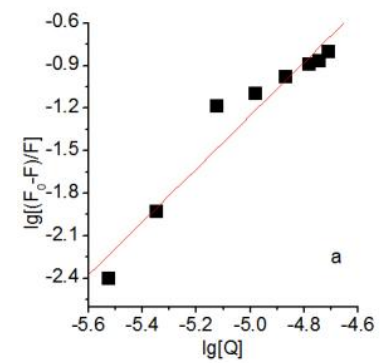

The relationship between fluorescence quenching intensity and the concentration of quenchers can be used to obtain the binding constant and the number of binding sites.

$$
\mathrm{Lg}(\mathrm{F} 0-\mathrm{F}) / \mathrm{F}=\mathrm{LgKb}+\mathrm{nLg}[\mathrm{Q}]
$$

The values of $\mathrm{Kb}$ and $\mathrm{n}$ at $303 \mathrm{~K}$ and $313 \mathrm{~K}$ were obtained from the double logarithm regression curve (Fig. 3).

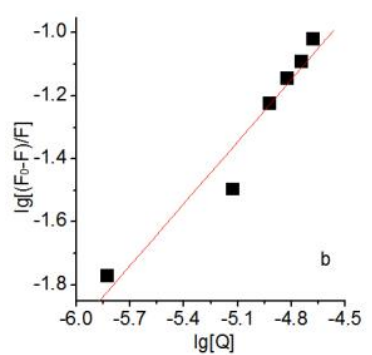

Figure 3. The plots of $\lg (\mathrm{F} 0-\mathrm{F}) / \mathrm{F}$ vs. $\lg [\mathrm{Q}]$ at different temperatures (a) $303 \mathrm{~K}$ and (b) $313 \mathrm{~K}$

The values of $\mathrm{Kb}$ and $\mathrm{n}$ at $303 \mathrm{~K}$ and $313 \mathrm{~K}$ are listed in Table II.

TABLE II. THE BINDING CONSTANT, KB, AND THE NUMBER OF BINDING SITES, N

\begin{tabular}{ccccc}
\hline $\mathrm{T} /(\mathrm{K})$ & double logarithm e quation & $\mathrm{R}$ & $K_{\mathrm{b}} /(\mathrm{L} / \mathrm{mol})$ & $\mathrm{n}$ \\
\hline 303 & $\operatorname{Lg}\left[\left(F_{0} / F\right) / F=8.1404+1.878 \mathrm{Lg}[\mathrm{Q}]\right.$ & 0.9670 & $1.3816 \times 10^{8}$ & 1.878 \\
313 & $\operatorname{Lg}\left[\left(F_{0} / F\right) / F=2.0043+0.657 \mathrm{Lg}[\mathrm{Q}]\right.$ & 0.9706 & $1.0010 \times 10^{2}$ & 0.657 \\
\hline
\end{tabular}


The $\mathrm{n}$ values are nearly 2 , and thus indicate the existence of two binding sites in BSA for EACHL. The interactions of EACHL with BSA decreased when rising the temperature, which suggest that interactions of EACHL with BSA were exothermic.

\section{H. Acting force}

The acting forces between drug and biomolecule are composed of weak interactions of molecules such as hydrogen bond formation, van der Waals forces, electrostatic forces, and hydrophobic interaction. The thermodynamic parameters could be calculated by equation (3), (4) and (5).

$$
\operatorname{Ln}(\mathrm{K} 2 / \mathrm{K} 1)=\Delta \mathrm{rHm}(1 / \mathrm{T} 1-1 / \mathrm{T} 2) / \mathrm{R}
$$

$\Delta \mathrm{rGm}=-\mathrm{RTLn}$

$\Delta \mathrm{rSm}=(\Delta \mathrm{rHm}-\Delta \mathrm{rGm}) / \mathrm{T}$

The thermodynamic parameters are presented in Table 3.

TABLE III. THERMODYNAMIC PARAMETERS FOR THE ASSOCIATION OF EACHL WITH HSA

\begin{tabular}{|c|c|c|c|}
\hline $\mathrm{T} / \mathrm{K}$ & $\Delta \mathrm{rHm} /\left(\mathrm{kJ} \cdot \mathrm{mol}^{-1}\right)$ & $\Delta \mathrm{rSm} /\left(\mathrm{J} . \mathrm{K}^{-1}\right)$ & $\Delta \mathrm{rGm} /\left(\mathrm{kJ} \cdot \mathrm{mol}^{-1}\right)$ \\
\hline 303 & & & -47.22 \\
\hline & -1175.425 & -4035.13 & \\
\hline 313 & & & -11.99 \\
\hline
\end{tabular}

As the table shows the values of the $\triangle \mathrm{rHm}, \triangle \mathrm{rSm}$ and $\triangle$ rGm are all negative numbers. The negative signs reveal that the binding processes are influenced by temperature, low temperature are spontaneous, high temperature are non-spontaneous. The negative $\Delta \mathrm{H}$ and $\Delta \mathrm{S}$ values suggest the van der Waals forces or hydrogen bond formation exist in EACHL and BSA.

\section{CONCLUSION}

The active ingredients (EACHL) were extracted from the Chinese honey locust. The Ic50 values of EACHL on the Eca109 and TE1 are $11.53,32.07 \mu \mathrm{g} \cdot \mathrm{ml}-1$, respectively. The interaction between EACHL and BSA has been investigated

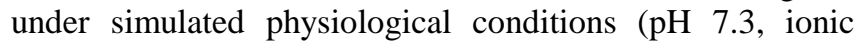
strength $0.1 \mathrm{~mol} / \mathrm{L}$ ) using fluorescence methodology. The constant $\mathrm{Kq}$ of the EACHL and BSA is larger than $2 \times 1010$ $\mathrm{L} \cdot \mathrm{mol}-1 \cdot \mathrm{s}-1$, which suggest that the fluorescence quenching mechanism of BSA by EACHL was consistent with static quenching. The binding reaction was influenced by temperature for $\Delta \mathrm{rHm}, \Delta \mathrm{rSm}$ and $\Delta \mathrm{rGm}$ is negative. $\Delta \mathrm{H}$ and $\Delta \mathrm{S}$ is negative suggest that Van der Waals forces or hydrogen bond play a major role in the EACHL-BSA interaction. ACKNOWLEDGMENTS

This research was supported by the special grade of the financial support from the China Postdoctoral Science Foundation (Grant No.2016T90669), the National Science Foundation for Post-doctoral Scientists of China (Grant No.2015M582189), the National Science Foundation (Grant No.81550014), the Henan Postdoctoral Sustentation Fund, China (Grant No. 00104256), Jinshui District science and technology bureau social program (Grant No. 20132602).

\section{REFERENCES}

[1] Jemal, F. Bray, M. Center, J. Ferlay, E. Ward, D. Forman, Global cancer statistics. CA:A Cancer Journal for Clincians. 61(2011) 69-90.

[2] F. C. Si, Inhibition of Qigesan and its separated prescriptions on angiogenesis in Esophageal carcinoma Eca109 cell transplanted mude mice tumor. World Chinese Journal of Digestology. 28 (2008)3139-3145.

[3] D. N. Oleynikov, A. V. Rohin, Galactomannan from the seeds of Chinese honey locust (Gleditsia sinensis Lam.). Applied Biochemistry and microbiology. 46(2010)103-107.

[4] Sankar, D. Sasanka, G. Shalini, G. Nikhil, Binding interaction between plasma protein bovine serum albumin and flexible charge transfer fluorophore: A spectroscopic study in combination with molecular docking and molecular dynamics simulation. Journal of Photochemistry and Photobiology A: Chemistry. 231(2012)19-27.

[5] S. M. S. Abdullah, S. Fatma, G. Rabbani, J. M. Ashraf, A spectroscopic and molecular docking approach on the binding of tinzaparin sodium with human serum albumin. Journal of Molecular Structure. 1127 (2017)283-288.

[6] R. Suparna, D.Somnath, P. Chiranjit, Chiranjit.Binding affinities of Schiff base Fe(II) complex with BSA and calf-thymus DNA: Spectroscopic investigations and molecular docking analysis. Spectrochimica acta. Part A. Spectrochimica acta. Part A, Molecular and biomolecular spectroscopy. 166(2016)84-94.

[7] Cao, D. Wu, H. Wang, M. Xu, Effect of the glycosylation of flavonoids on interaction with protein. Spectrochimica acta. Part A, Molecular and biomolecular spectroscopy. 73(2009) 972-975.

[8] F. Cui, Y. Yan, Q. Zhang, X. Yao, G. Qu, Y. Lu, Characterization of the interaction between 8-bromoadenosine with human serum albumin and its analytical application. Spectrochimica acta. Part A, Molecular and biomolecular spectroscopy. 74 (2009)964-971.

[9] F. Y. Zhang, Y. N. Ni, A Comparison Study on the Interaction of Sunset Yellow and $\beta$-Carotene with Bovine Serum Albumin Acta Chimica Sinica. 12 (2012)59-64. 